\title{
Advanced Data Delivery Strategy Based on Multiperceived Community with IoT in Social Complex Networks
}

\author{
Jingwen Luo, Jia Wu (D), and Yuzhou Wu \\ School of Computer Science and Engineering, Central South University, Changsha 410083, China \\ Correspondence should be addressed to Jia Wu; jiawu5110@163.com
}

Received 4 November 2019; Revised 25 December 2019; Accepted 18 January 2020; Published 11 February 2020

Guest Editor: Yuan Yuan

Copyright (c) 2020 Jingwen Luo et al. This is an open access article distributed under the Creative Commons Attribution License, which permits unrestricted use, distribution, and reproduction in any medium, provided the original work is properly cited.

\begin{abstract}
With the advent of $5 \mathrm{G}$ communication standards, the number of $5 \mathrm{G}$ base stations increases steadily, and the number of mobile terminals and IoT (Internet of Things) devices increases sharply, which sharps a large number of IoT devices and forms a complex network. These devices can take as nodes of a community in the opportunistic social network. However, in the environment of traditional opportunistic network algorithm and mass data transmission, information transmission is only carried out at several source nodes in the community, which usually leads to transmission delay, excessive energy consumption, and source node death. Therefore, we propose an effective data delivery based on the multiperceived domain algorithm, which recombines communities based on the correlation degree of nodes, and new communities assist source nodes to transmit information in solving these problems. The comparison between the experiment and the classical opportunistic network algorithm shows that the method has outstanding performance in reducing the resource consumption of data transmission and improving the efficiency of information transmission.
\end{abstract}

\section{Introduction}

In the rapidly developing mobile industry in recent years, the network infrastructure has enough network bandwidth to meet the communication of Internet connection equipment. From basic sensors to extremely complex cloud servers, these devices constitute the Internet of Things [1-3]. Moreover, the rapid proliferation of personal mobile devices creates abundant opportunities to transmit data for mobile networks. An in-depth study has been started on a mobile network, which is an opportunistic network that does not need to establish a complete end-to-end communication path between a source node and a destination node $[4,5]$. It is a type of complex network that dynamically selects adjacent mobile devices as neighbor nodes to construct the communication path based on the "Storage-Carrying-Forwarding" mechanism [6].

Along with the advent of the $5 \mathrm{G}$ era of communication, an increasing number of Internet services are migrating to the mobile infrastructure or devices, such as cell phones, tablet computers, smart watches, UAV (unmanned aerial vehicles) [7], IoV (Internet of connected vehicles) [8], and intelligent vehicle devices [9-11]. These devices carried by people have the characteristics of random movement and certain human society. It can be regarded as a social node, and the community can be established through the correlation of nodes [12]. In social networks, nodes with a high correlation with others may transmit a large amount of data [13]. With the rise of the Internet of Things (IoT), the author puts forward a Social Internet of Things (SIoT) that integrates the concept of social network into the solution of the Internet of Things [14]. The burden on the communication network can be relieved by neighboring devices transmitting information in the social network $[15,16]$.

Mobile devices carried by people and IoT devices installed in all corners can realize fast and large-capacity data transmission requirements under $5 \mathrm{G}$ communication standard, thus giving rise to the growing demand for fast and large-capacity data transmission [17-19]. For traditional algorithms, many communities deliver information depending on one or two source nodes in the social complex networks. These nodes not only transmit big volume of data 
but also require to calculate some tasks, which need more energy consumption [12, 20-23]. In traditional opportunistic network protocols, if the source node does not have sufficient cache or overhead, it may lead to a long waiting time for data transmission in the community [24]. Even worse, these nodes will consume a lot of resources to transmit information based on flooding technology and lose data due to the death of the source node.

To solve the abovementioned problems, this paper proposes an effective data delivery scheme based on multiperceived community. The community is reconstructed according to the degree of association between the source node and the neighboring nodes. Some of the data transmission tasks of the source node are undertaken by the nodes of the new community. It can reduce the consumption of source node and prolong the life cycle of source node.

The contributions of this research study are as follows:

(1) For the reconstruction of the community, the source nodes need to carry out the reconstruction according to the association degree of the nodes, and the information transmission also needs to select the appropriate community.

(2) The algorithm offered by this paper can effectively deliver information and reduce the consumption of source nodes in the process of data transmission.

(3) Experiments show that this method has excellent performance in reducing energy consumption and improving data delivery efficiency.

The rest of the paper is arranged as follows: The second section describes the research on opportunistic networks. On this basis, we put forward the model of community and data transmission in the third section. Then, in the fourth section, the performance of the model is verified through simulation experiments. At the end of the article, the full text is summarized.

\section{Related Work}

To date, the opportunistic network research has tended to focus on routing algorithms. Various routing algorithms have been proposed in the last decade, and several routing protocols used in opportunistic networks are described as follows.

Vahdat and Becker [25] proposes the Epidemic routing algorithm, and the core idea of Epidemic is using a couple of encounter nodes to transmit information. These nodes deliver the information copy to the next meeting nodes. The Epidemic routing algorithm achieves $100 \%$ data delivery, but it adopts flooding techniques to deliver information, which sharply increases the network overhead. Lu et al. [26] proposed an energy-saving n-epidemic routing protocol theory, which can reduce the chance of nodes forwarding packets to neighboring nodes, thus improving the data transmission performance of basic epidemic routing protocols by more than four times. Guan and $\mathrm{Wu}$ [27] states a store-and-forward mechanism epidemic algorithm, which can deliver information, and can be guaranteed to be the shortest by increasing bandwidth and buffering memory space.

Burgess et al. [28] proposes the MaxProp routing protocol. Its core idea is to take precedence to schedule delivery and drop packets in a limited storage and bandwidth. The MaxProp routing algorithm avoids each message transmission using the flooding technique in the whole network and reduces routing overhead. Das et al. [29] recommends a Time-To-Live (TTL) based on the MaxProp routing algorithm, which based on hop count and TTL values prioritize the schedule of packets in data transmission. Compared with the MaxProp algorithm, this algorithm can greatly increase the amount of information transmission and reduce the overhead rate and delay under the same network resource consumption.

Spyropoulos et al. [30] offers the Spray and Wait routing algorithm, which is based on the flooding technique of twohop relay. The algorithm contains two parts, which are the source node sprays a set number of data copies to the network in the Spray stage, and the nodes directly deliver information to the destination node in the waiting stage. Wang et al. [31] proposed a dynamic Spray and Wait routing algorithm, which can make the number of copies of information be sent in a dynamic manner, thus improving transmission efficiency and transmission probability and reducing overhead and delay. Guan et al. [32] proposes a social relationship based on the adaptive multiple Spray and Wait routing algorithm. It can enhance the data delivery ratio and decline the information dwell time in the cache that improves the buffer effectively.

Lindgren et al. [33] designs a probabilistic routing protocol for intermittently connected networks called PRoPHET, which is able to transmit more data with a lower routing overhead. Xue et al. [34] recommends an advanced PRoPHET routing algorithm, which uses average delivery predict abilities to transmit information and avoid routing jitters. Han et al. [35] proposes an improved PRoPHET routing algorithm in the delay tolerant network (DTN). It appropriately selects the threshold of forwarding counter and hops counter that are able to improve the transmission probability, average delay, and overhead ratio.

Musolesi and Mascolo [36] designs the context-aware adaptive routing (CAR) protocol. The CAR routing algorithm adopts the Kalman filter for selecting the next hop, and each message only generates one copy, which saves cache and network resources. Niu et al. [37] recommends the Predict and Spread (PreS) routing protocol, which adopts the Markov chain for the node mobility model to achieve the social characteristics of nodes. Although the PreS algorithm has good performance in terms of delivery rate and delivery delay, because it does not consider node communication between different venues, nodes only deliver information on the same main venue.

Based on the above methods and problems, we can find a more effective method to solve the problems related to improving data transmission efficiency and reducing energy consumption in the complex social network. 


\section{Model Design}

3.1. Community Model Design. In a large data transmission environment, the death rate of source nodes is remarkable in some classical communication strategies. For instance, a communication domain may contain lots of nodes, but only including one or two source nodes, and the source node is required to send messages to each communication domain at node movement, which is a complex work. This communication network becomes a complex system. The ineffective information transmission causes the enormous consumption of source nodes and source node death, and data carried by the source node are lost in the end.

To avoid the overconsumption of the nodes' resource and reduce the rate of node death, we need to search some appropriate adjacent nodes assisting the source node in delivering a part of data. Therefore, we design an improved community model based on the process of communication domain information transmission.

Figure 1 is an illustration of a communication domain transferring information in a mass data transmission environment, in which both source nodes and neighboring nodes are included. We define that the source node $S$ has $n$ adjacent nodes at $t$ moment and $n \subseteq N$. $N$ is the number of the current network's nodes.

In order to reduce the resource consumption of the source node and avoid node death, we need to search for a node with high correlation with the source node $S$ and name it as an available node. Thus, the definition of the available node can depend on the correlation degree of the source node $\mathrm{S}$ and the adjacent node $A_{i}$, and it must be satisfied with the condition $\alpha \omega_{s i} \geq \theta$. $\alpha \omega_{s i}$ represents the correlation between the source node $S$ and the adjacent node $A_{i}, \alpha$ is a coefficient, $\omega_{s i}$ is the weight between the source node $S$ and the adjacent node $A_{i}$, and $\theta$ is the threshold.

As shown in Figure 2, some adjacent nodes match the condition $\alpha \omega_{s i} \geq \theta$, and they turn to available nodes. These available nodes can also communicate with the source node when receiving information from the source node. At the same time, they can also transmit information to other nodes and reduce the consumption of the source node.

In the opportunistic network, nodes usually are mobile devices that adopt a form of "store-carry-forward" to communicate with each other. It has randomness and mobility features. We need to analyse the relationship between nodes and consider establishing an effective mobility community model. Moreover, we intend to prove the variation of communities in the process of source node movement. The deduction and proof are as follows.

According to weighted networks in complex networks [38], we can define the structure degree of community $X$ is

$$
\Phi_{x}=\frac{\kappa_{x}}{\kappa}-\frac{\varepsilon_{s}^{2}}{(2 \kappa)^{2}}=\frac{4 \kappa \kappa_{x}-\varepsilon_{s}^{2}}{(2 \kappa)^{2}},
$$

where $\Phi_{x}$ is the structure degree of community $X, \kappa$ is the total weight in the communication networks, $\kappa_{x}$ is the total weight of community $X, \varepsilon_{s}$ is the total degree of source node
S community, $\Delta \omega$ is the variation of weight, $\omega_{s i}$ is the edge weight between source node $S$ and adjacent node $A_{i}$, and $\epsilon_{X}^{s}$ is the edge weight in source node $S$ in community $X$.

Deduction 1. In the process of the source node $S$ movement, if it satisfied the condition $\left(\varepsilon_{s} \varepsilon_{i} / 2 \kappa\right)<\omega_{s i}<\left(\varepsilon_{s} \varepsilon_{i}+\varepsilon_{s} \Delta \omega+\right.$ $\left.\Delta \omega^{2} / 2(\kappa+\Delta \omega)\right)+\Delta \omega$, the source node $S$ will be separated from the community of node $A_{i}$.

Proof 1 . The assumption here is that the community $X$ has separated into two subcommunities $C_{x 1}$ and $C_{x 2}$ : the source node $S$ in community $X 1$ and node $A_{i}$ in community $X 2$, and the total weight of the network has decreased. Then,

$$
\left\{\begin{array}{l}
\kappa_{x 1}+\kappa_{x 2}<\kappa, \\
\frac{4 \kappa \kappa_{x 1}-\varepsilon_{s}^{2}}{(2 \kappa)^{2}}+\frac{4 \kappa \kappa_{x 2}-\varepsilon_{i}^{2}}{(2 \kappa)^{2}}<\frac{4 \kappa\left(\varepsilon_{s}+\varepsilon_{i}+\omega_{s i}\right)-\left(\varepsilon_{s}+\varepsilon_{i}\right)^{2}}{(2 \kappa)^{2}} \\
\omega_{s i}>\frac{\varepsilon_{s} \varepsilon_{i}}{2 \kappa}
\end{array}\right.
$$

The total weight has decreased, and the above formula can be shown as follows:

$$
\left\{\begin{array}{l}
\kappa_{x 1}^{\prime}+\kappa_{x 2}^{\prime}>\kappa^{\prime}, \\
\omega_{s i}<\frac{\varepsilon_{s} \varepsilon_{i}+\varepsilon_{s} \Delta \omega+\Delta \omega^{2}}{2(\kappa+\Delta \omega)}+\Delta \omega .
\end{array}\right.
$$

Therefore, the community $X$ have separated into two subcommunities $C_{x 1}$ and $C_{x 2}$, which satisfied the condition $\left(\varepsilon_{s} \varepsilon_{i} / 2 \kappa\right)<\omega_{s i}<\left(\varepsilon_{s} \varepsilon_{i}+\varepsilon_{s} \Delta \omega+\Delta \omega^{2} / 2(\kappa+\Delta \omega)\right)+\Delta \omega$.

Deduction 2. In the process of the source node $\mathrm{S}$ movement, the edge of the source node $\mathrm{S}$ connected node $A_{i}$ and the edge is the only edge of node $A_{i}$. If the weight between node $\mathrm{S}$ and node $A_{i}$ has declined, the node $A_{i}$ will not separate from the community.

Proof 2. Similar to deduction 1 , the assumption that the reduction of edge weight between source node $S$ and node $A_{i}$ is $\Delta w, \Delta w<0$. If the community $X$ has separated, that must satisfy the condition as follows:

$$
\left\{\begin{array}{l}
\kappa_{x 1}+\kappa_{x 2}<\kappa, \\
\frac{4 \kappa \kappa_{x 1}-\varepsilon_{s}^{2}}{(2 \kappa)^{2}}+\frac{4 \kappa \kappa_{x 2}-\varepsilon_{i}^{2}}{(2 \kappa)^{2}}<\frac{4 \kappa\left(\varepsilon_{s}+\varepsilon_{i}+\omega_{s i}\right)-\left(\varepsilon_{s}+\varepsilon_{i}\right)^{2}}{(2 \kappa)^{2}}, \\
\omega_{s i}>\frac{\varepsilon_{s} \varepsilon_{i}}{2 \kappa} .
\end{array}\right.
$$

Then, 

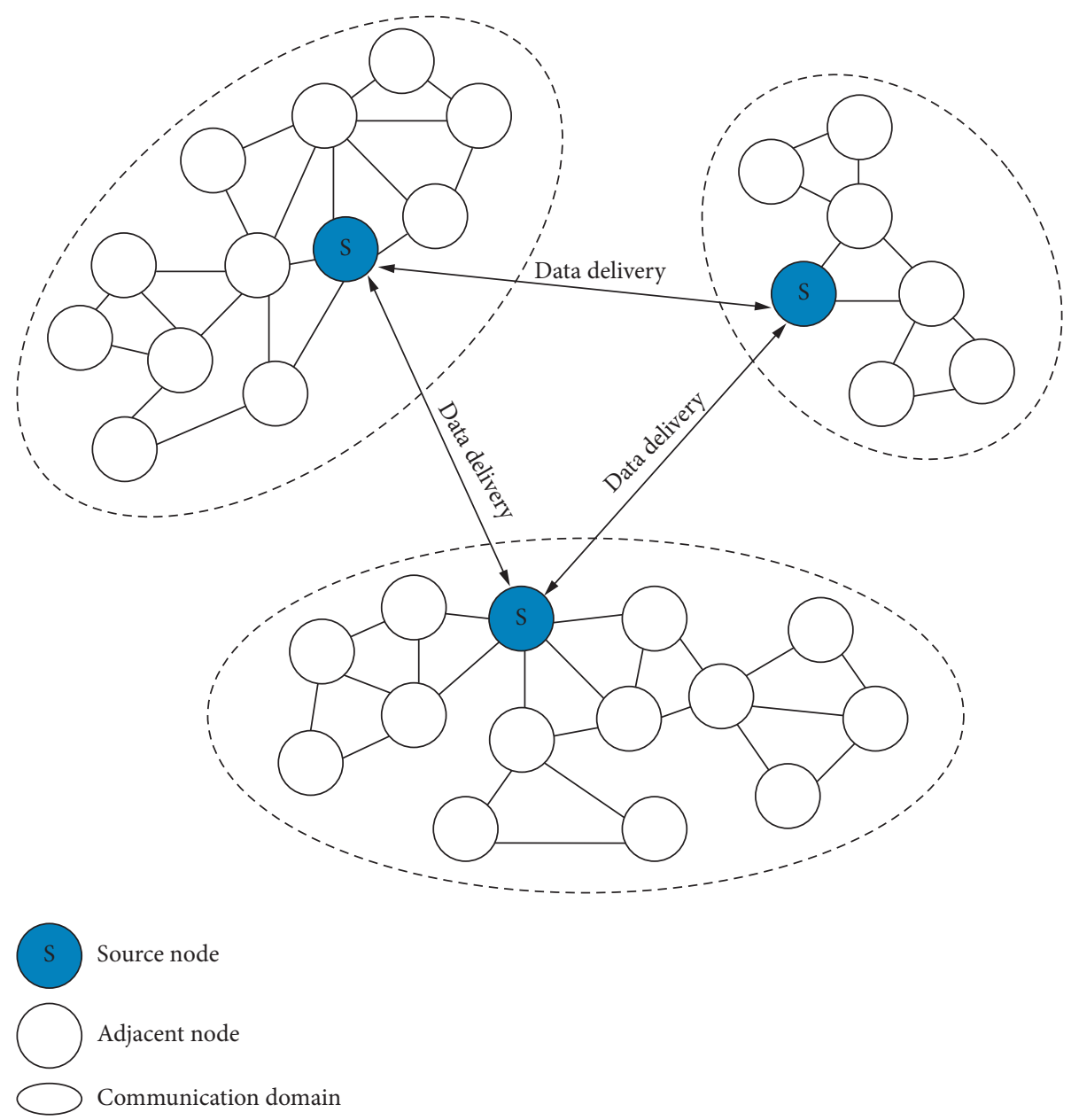

Figure 1: Data delivery on communication domain.

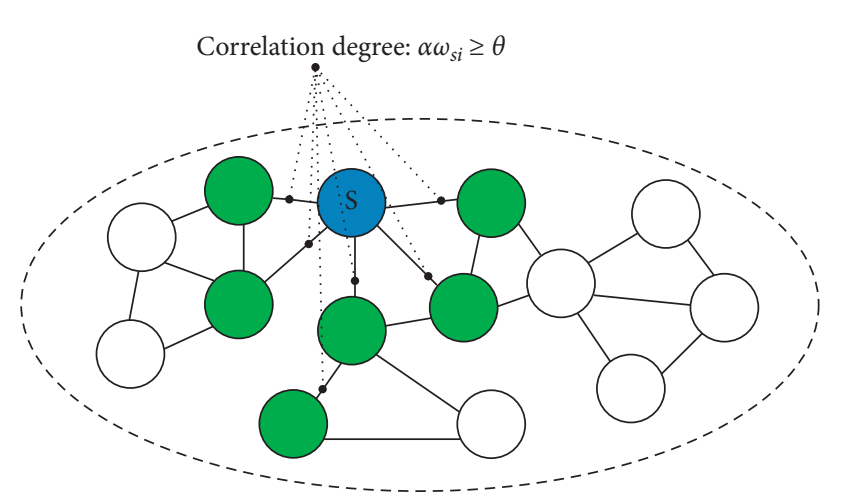

Source node

Adjacent node

Available node

FIgURE 2: Searching available nodes.

$$
\left\{\begin{array}{l}
\kappa_{x 1}^{\prime}+\kappa_{x 2}^{\prime}>\kappa \prime \\
\omega_{s i}<\frac{\varepsilon_{s} \varepsilon_{i}+\varepsilon_{s} \Delta \omega+\Delta \omega^{2}}{2(\kappa+\Delta \omega)}+\Delta \omega .
\end{array}\right.
$$

That is,

$$
\frac{\varepsilon_{s} \varepsilon_{i}}{2 \kappa}<\omega_{s i}<\frac{\varepsilon_{s} \varepsilon_{i}+\varepsilon_{s} \Delta \omega+\Delta \omega^{2}}{2(\kappa+\Delta \omega)}+\Delta \omega .
$$

It can be explained as

$$
\frac{\varepsilon_{s} \varepsilon_{i}}{2 \kappa}<\omega_{s i}<\frac{\varepsilon_{s} \varepsilon_{i}+\varepsilon_{s} \Delta \omega}{2(\kappa+\Delta \omega)} .
$$

Because

$$
\frac{\varepsilon_{s} \varepsilon_{i}+\varepsilon_{s} \Delta \omega}{2(\kappa+\Delta \omega)}-\frac{\varepsilon_{s} \varepsilon_{i}}{2 \kappa}=\frac{\varepsilon_{s} \Delta \omega\left(\kappa-\varepsilon_{i}\right)}{2 \kappa(\kappa+\Delta \omega)}<0 .
$$

We can get that $\left(\varepsilon_{s} \varepsilon_{i} / 2 \kappa\right)<\omega_{s i}<\left(\varepsilon_{s} \varepsilon_{i}+\varepsilon_{s} \Delta \omega / 2(\kappa+\Delta \omega)\right)$ is a false proposition. 
Thus, the only edge of node $A_{i}$ connects the source node $\mathrm{S}$ and its weight decline, and the node $A_{i}$ will not separate from the community.

Deduction 3. The source node $\mathrm{S}$ in community $X$, its edge weight, and the weight of community $Y$ have been increased, which satisfied the condition $4(\kappa+\Delta \omega)\left(\varepsilon_{Y}^{s}-\varepsilon_{X}^{s}+\right.$ $\Delta \omega)+\left(\varepsilon_{s}+\Delta \omega\right)\left(\varepsilon_{X}-\varepsilon_{Y}-\varepsilon_{s}\right)-\Delta \omega^{2}>0$; the source node $S$ can join in community $Y$.

Proof 3. The edge weight of community $X$ and $Y$ has risen, and the structure degree of communities is

$$
\Phi_{x}+\Phi_{y}=\frac{2 \kappa \kappa_{x}-\left(\varepsilon_{x}+\Delta \omega\right)^{2}}{4(\kappa+\Delta \omega)^{2}}+\frac{2 \kappa \kappa_{y}-\left(\varepsilon_{y}+\Delta \omega\right)^{2}}{4(\kappa+\Delta \omega)^{2}} .
$$

When the source node $S$ leaves community $X$ and joins in community $Y$, the structure degree of communities is

$$
\begin{aligned}
\Phi_{x-s}+\Phi_{y+s}= & \frac{4 \kappa\left(\kappa_{x}-\epsilon_{X}^{s}\right)-\left(\varepsilon_{x}+\Delta \omega\right)^{2}}{4(\kappa+\Delta \omega)^{2}} \\
& +\frac{4 \kappa \kappa_{y}-\left(\varepsilon_{y}+\Delta \omega\right)^{2}}{4(\kappa+\Delta \omega)^{2}} .
\end{aligned}
$$

To prove that the structure degree of community $Y$ will increase if source node $S$ joins in, then it requires to prove $\Phi_{x-s}+\Phi_{y+s}>\Phi_{x}+\Phi_{y}$. That is,

$4(\kappa+\Delta \omega)\left(\epsilon_{Y}^{s}-\epsilon_{X}^{s}+\Delta \omega\right)+\left(\varepsilon_{s}+\Delta \omega\right)\left(\varepsilon_{X}-\varepsilon_{Y}-\varepsilon_{s}\right)-\Delta \omega^{2}>0$.

Through the above deduction, we have proved several changes in the community during the movement of the source node. In the next section, we will use the degree of association of nodes to divide a community and recombine multiple communities to transfer data.

3.2. Data Delivery Model Design. In the process of data transmission of the opportunistic network, information delivers to destination nodes requiring multiple hop transmission. In addition, when the traditional communication strategy sends complete data to each node in the community, it is usually done in an overlay manner. It will cause more significant routing overhead and energy consumption. In addition, there are many fake nodes or honeypot nodes in the social network. The fake nodes or honeypot nodes can leak information in the data transmission process, and it is a potential security problem.

To reduce the risk of information leakage and save source nodes' routing overhead and energy consumption, we design a data delivery based on multiperceived domain (DDMPD) scheme to solve these problems.

In Figure 3, the source node $\mathrm{S}$ communicates with available nodes, and the available node has received and stored a part of data from the source node $S$, which transformed into a relay node. Relay nodes can widely transmit information to other nodes. Whenever the source node moves, it searches for available nodes from neighboring nodes and sends a message to convert some appropriate available nodes into relay nodes.
Recombining the communication domain after relay nodes have turned, and it reconstructed the old community into several new communities. The pressure on the source node to transmit data and spread more information can be relieved through these new communities and ensure that data are transmitted on the line.

To describe the information delivery process clearly, the work flow of the DDMPD scheme is as follows:

Step 1. We denote the total data carried by the source node $S$ as $D_{0}(\varphi)=\varphi_{0}$, and the source node sends a "HELLO" message to adjacent nodes that search for available nodes of a community.

Step 2. When the appropriate available node receives a "HELLO" message in the community, the node will respond and establish a link with the source node and convert it into a relay node.

Step 3. The source node starts communicating with the relay node and transmits half data to relay nodes of the multiperception community. When the source node marks that data have been transmitted, the relay node of community 1 receives information from the source node $S$ that completed the first data transmission (Figure 4).

Then, the information carried by community 1 is as follows:

$$
D_{1}(\varphi)=\frac{1}{2} D_{0}(\varphi)=\frac{1}{2} \varphi_{0}
$$

The unsent surplus data of the source node $S$ after firsttime transmission are $D_{\text {surl }}(\varphi)$

$$
D_{\text {surl }}(\varphi)=D_{0}(\varphi)-D_{1}(\varphi)=\varphi_{0}-\frac{1}{2} \varphi_{0}=\frac{1}{2} \varphi_{0} .
$$

Step 4. The source node continues to move, and it sends a "HELLO" message and searches for available nodes of the community.

Step 5. When the source node establishes communication with the multiperception community 2 , the source node sends half unsent surplus data to nodes of community 2 . In addition, there is data transmission between communities, and the nodes of community 1 broadcast half of the information received from the source node (Figure 5).

Community 2 received the following information:

$$
\begin{aligned}
D_{2}(\varphi) & =\frac{1}{2} D_{\text {surl }}(\varphi)+D_{1}(\varphi)=\frac{1}{2^{2}} D_{0}(\varphi)+D_{1}(\varphi) \\
& =\frac{1}{2^{2}} \varphi_{0}+\frac{1}{2^{1}} \varphi_{0}=\frac{3}{4} \varphi_{0} .
\end{aligned}
$$

The unsent surplus information of source node $S$ after second-time transmission is as follows:

$$
D_{\text {sur2 }}(\varphi)=D_{0}(\varphi)-D_{2}(\varphi)-D_{1}(\varphi) \text {. }
$$

We know that the data of community 2 contains all data received from community 1 , which is $D_{1}(\varphi) \subseteq D_{2}(\varphi)$. Thus, 


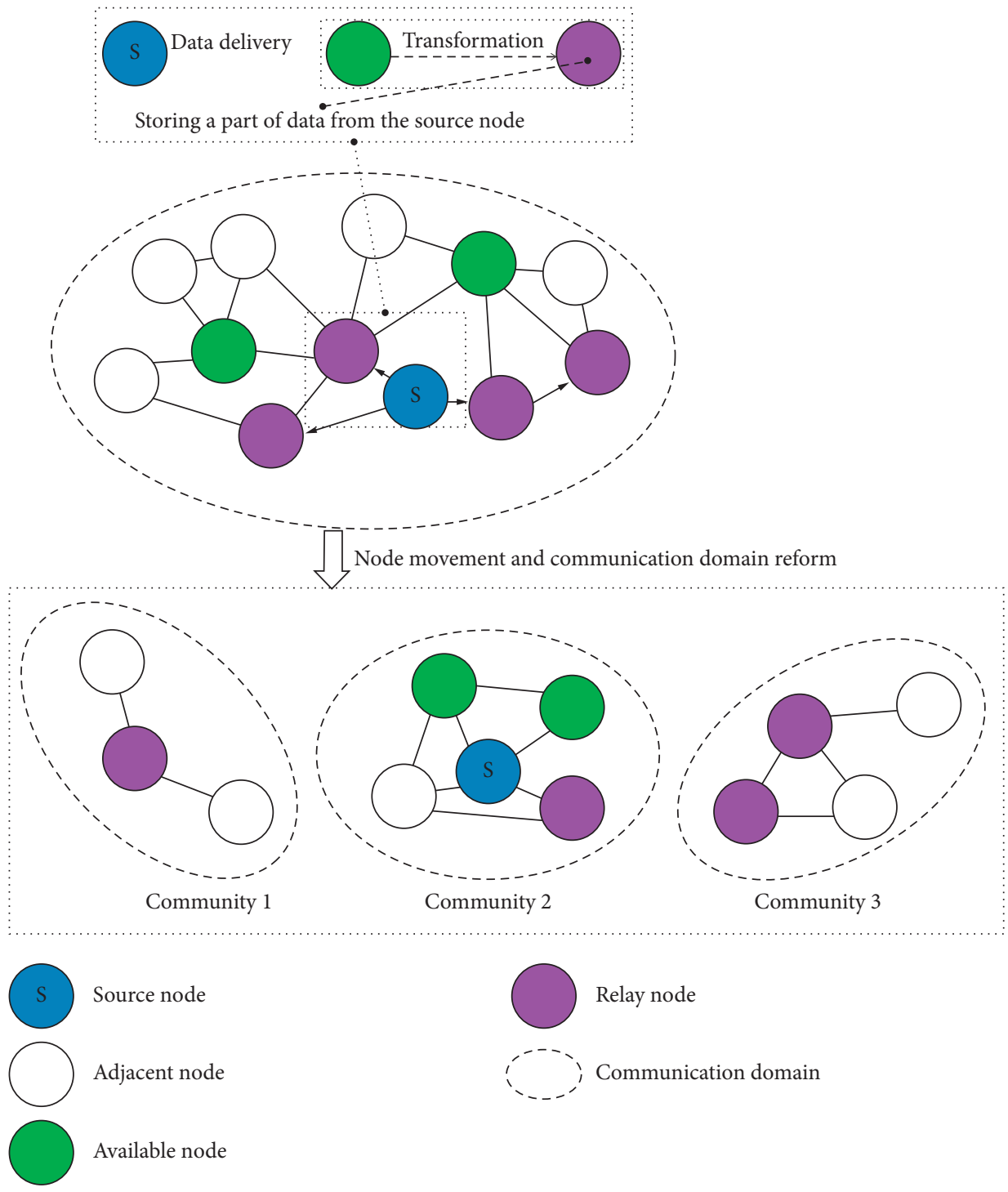

FIgURE 3: The process of data transmission and communication domain reform.

the unsent surplus information of source node $S$ after second-time transmission is as follows:

$$
D_{\text {sur } 2}(\varphi)=D_{0}(\varphi)-D_{2}(\varphi)=\varphi_{0}-\frac{1}{2^{2}} \varphi_{0}-\frac{1}{2^{1}} \varphi_{0}=\frac{1}{4} \varphi_{0} .
$$

The source node marks the unsent surplus information and continues to move.

Step 6. Assuming it could communicate between communities so community 3 could receive the message from communities 1 and 2, when the source node encounters the perception community 3 and starts information transmission. The information received by community 3 is as follows:

$$
\begin{aligned}
D_{3}(\varphi) & =\frac{1}{2} D_{\text {sur } 2}(\varphi)+D_{2}(\varphi)+D_{1}(\varphi)=\frac{1}{2^{3}} D_{0}(\varphi)+D_{2}(\varphi) \\
& =\frac{1}{2^{3}} \varphi_{0}+\left(\frac{1}{2^{2}}+\frac{1}{2^{1}}\right) \varphi_{0}=\frac{7}{8} \varphi_{0} .
\end{aligned}
$$

The source node marks the surplus information and continues to move. The unsent surplus information of source node $\mathrm{S}$ after the third-time transmission is as follows:

$$
D_{\text {sur } 3}(\varphi)=D_{0}(\varphi)-D_{3}(\varphi)=\varphi_{0}-\frac{1}{2^{3}} \varphi_{0}-\frac{1}{2^{2}} \varphi_{0}-\frac{1}{2^{1}} \varphi_{0}=\frac{1}{8} \varphi_{0} .
$$

Step 7. Repeat the procedure described above. 


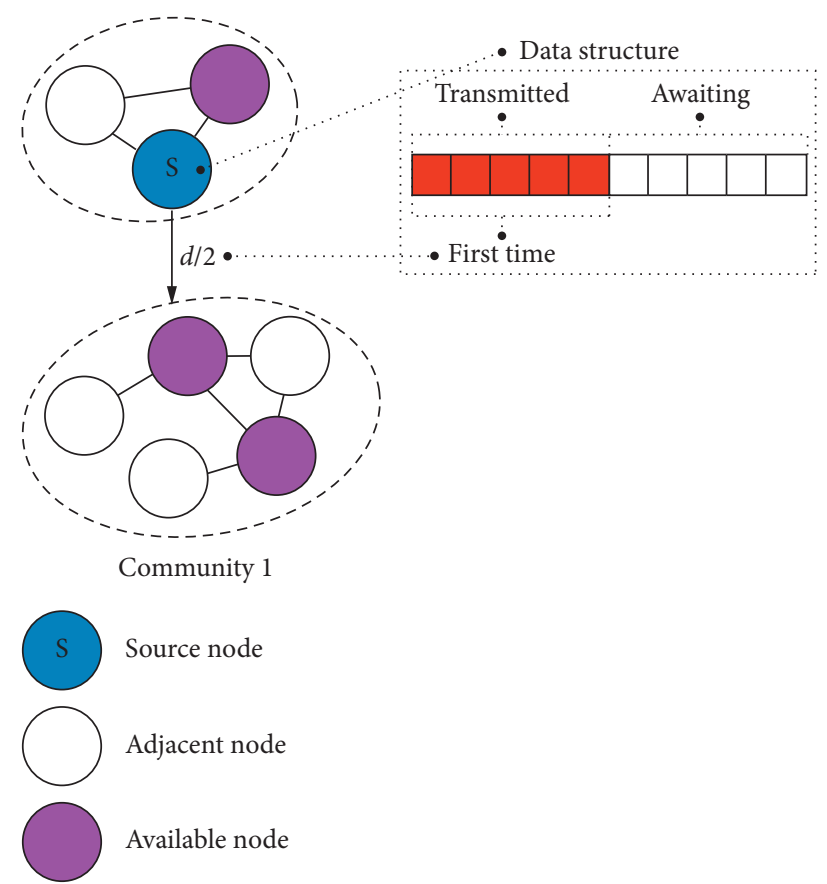

FIGURE 4: Information delivery and data structure in first-time transmission.

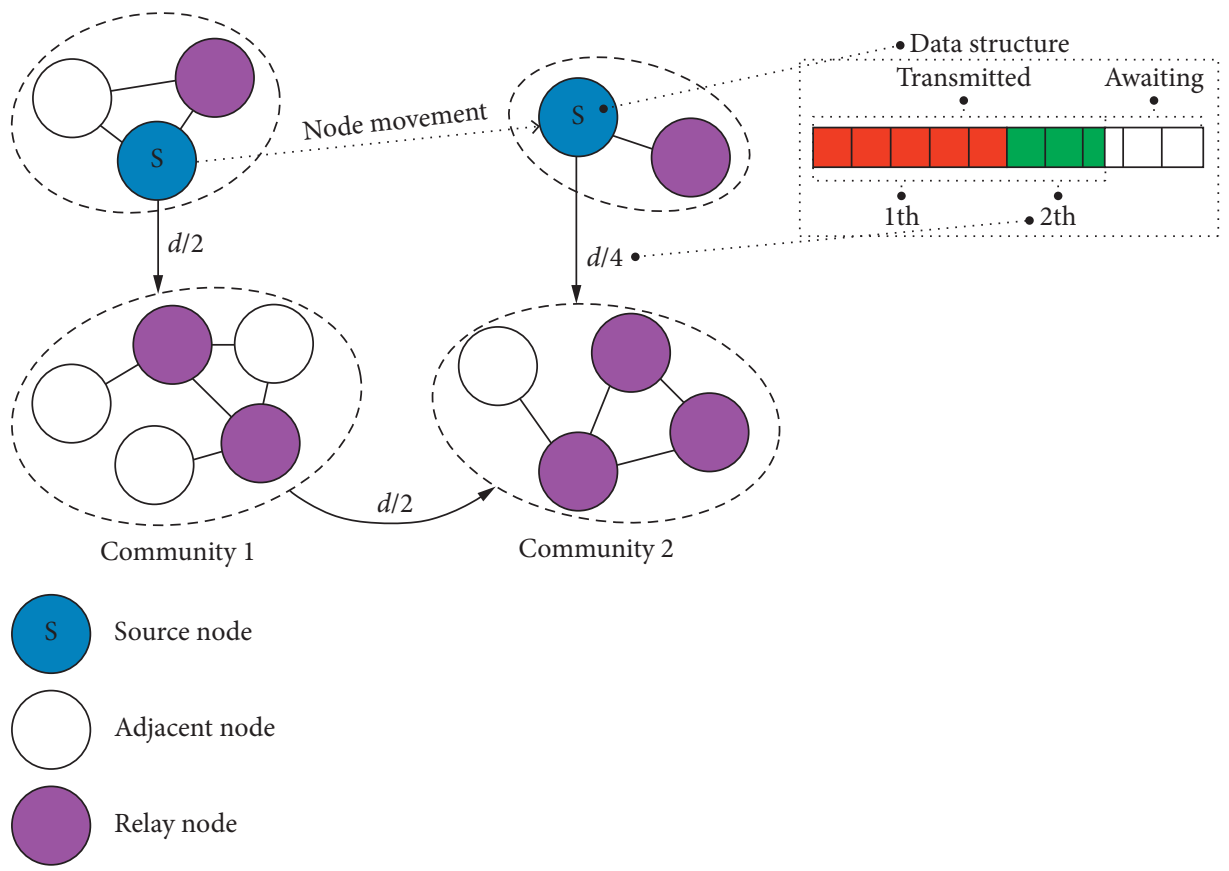

FIGURE 5: Information delivery and data structure in second-time transmission.

Step 8. When the source node searches for the community of the target node after $n$ times of information transmission, then $\left(1 / 2^{n}\right) D_{0}$ is the community information of the target node of the source node, and the data received by the communication between communities are $\sum_{k=1}^{n-1} D_{k}(\varphi)$; the whole transmission process is indicated in Figure 6.

Therefore, the following information is received by the community of destination nodes:

$$
\begin{aligned}
D_{n}(\varphi) & =\frac{1}{2} D_{\operatorname{sur}(n-1)}(\varphi)+\sum_{k=1}^{n-1} D_{k}(\varphi)=\frac{1}{2^{n}} D_{0}(\varphi)+D_{n-1}(\varphi) \\
& =\frac{1}{2^{n}} \varphi_{0}+\left(\frac{1}{2^{n-1}}+\frac{1}{2^{n-2}}+\cdots+\frac{1}{2^{1}}\right) \varphi_{0}=\left(1-\frac{1}{2^{n}}\right) \varphi_{0} .
\end{aligned}
$$




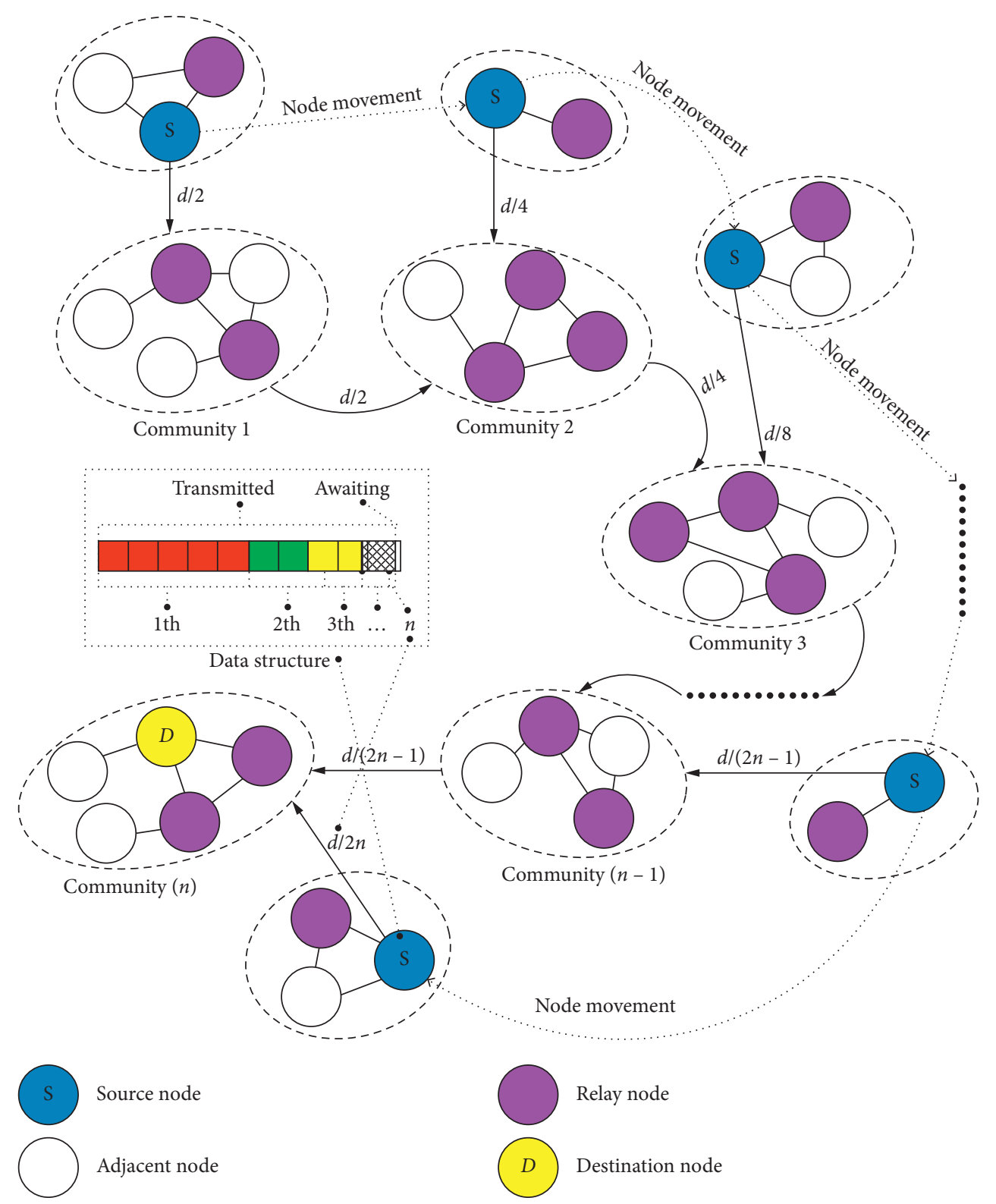

FIgURe 6: Process of the source node deliver information and data structure in $n$-time transmission.

By the above equation, due to more transmissions that the destination node receives, more data closer to the complete information is carried by the source node.

We can see that only a small part of the information is waiting, and most of the information has been delivered to the community and target nodes in Figure 6 . The unsent information of source is

$$
\begin{aligned}
D_{\operatorname{sur}(n)}(\varphi) & =D_{0}(\varphi)-\sum_{k=1}^{n} D_{k}(\varphi)=D_{0}(\varphi)-D_{n}(\varphi) \\
& =\varphi_{0}-\left(1-\frac{1}{2^{n}}\right) \varphi_{0}=\frac{1}{2^{n}} \varphi_{0} .
\end{aligned}
$$

When $n \longrightarrow \infty$, the information quantity transmitted by the DDMPD scheme is equal to the Epidemic and Spray and Wait routing algorithm. The longer the information transmission time, the more complete the information received by the destination node. From above, we can establish an algorithm to explain this scheme (Algorithm 1).

According to the above DDMPD algorithm, the destination node needs the source node to send $n$ times of information to search for, and $n-1$ communities also need to participate in the data transmission. The source node and communities adopt the parallel transmission method to transmit information. Therefore, DDMPDs time complexity is $O(n+(n-1))=O(2 n-1)=O(n)$. As compared to the classical opportunistic network routing algorithm Epidemic and Spray and Wait, the time complexity of Epidemic and Spray and Wait is $O(n 2)$ and $O(n)$, respectively. Generally speaking, DDMPD has the same time complexity as Spray and Wait's, but its time complexity is lower than Epidemic. 


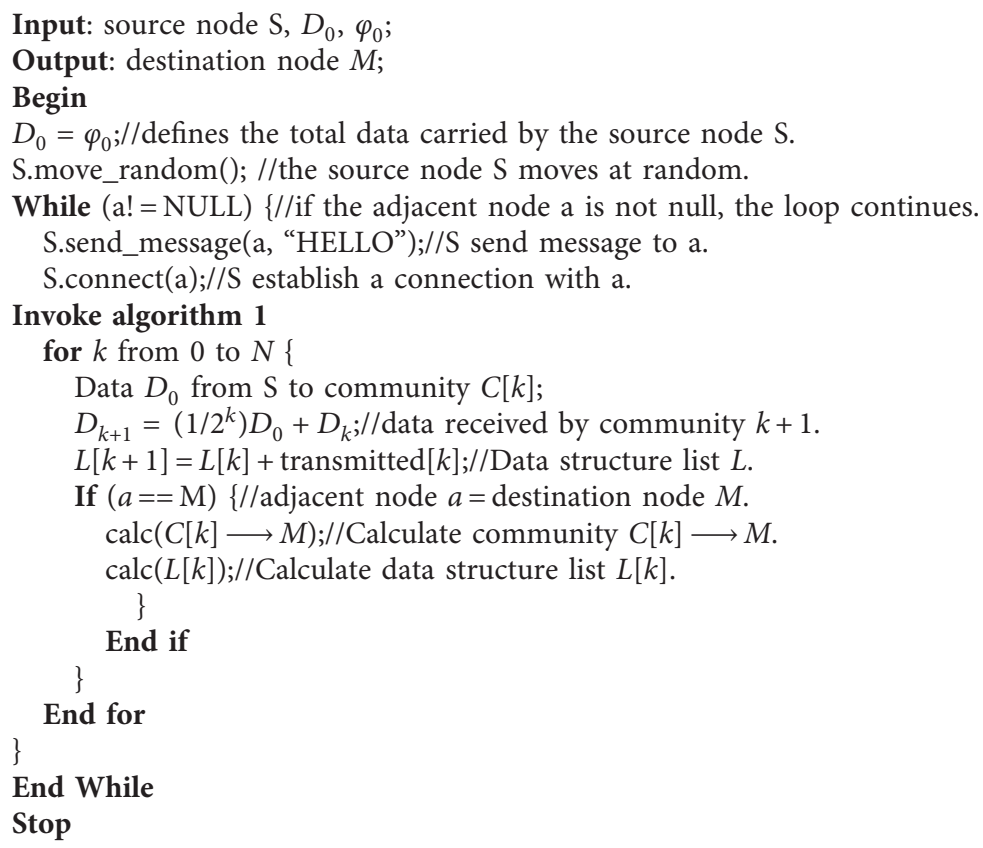

Algorithm 1: The data delivery based on multiperceived domain (DDMPD) algorithm.

In the DDMPD scheme, the source node delivers $\left(1 / 2^{1}\right) D_{0}$ data to the next community in the first-time transmission, and it transmits $\left(1 / 2^{2}\right) D_{0}$ data to the next community in the second time. After $n-1$ and $n$ transmission, the source node's data transmission is $\left(1 / 2^{n-1}\right) D_{0}$ and $\left(1 / 2^{n}\right) D_{0}$, respectively. Therefore, we can calculate the total routing overhead of DDMPD is $\mathrm{RO}_{\mathrm{DDMPD}}=$ $\sum_{k=1}^{n}(1 / 2)^{k}=1-(1 / 2)^{n}$. The complete data need the source node to transmit to other communities. After $n$ times of data transmission, $\mathrm{RO}_{\text {Epidemic }}=n$ and $\mathrm{RO}_{\text {Spray and Wait }}=n$ are the routing overhead of Epidemic and Spray and Wait. Through the above analysis, we can figure out the proportion of these algorithms as follows:

$$
\frac{\mathrm{RO}_{\text {DDMPD }}}{\mathrm{RO}_{\text {Epidemic }}}=\frac{\mathrm{RO}_{\text {DDMPD }}}{\mathrm{RO}_{\text {Spray and Wait }}}=\frac{1-(1 / 2)^{n}}{n} \text {. }
$$

According to formula (21), the routing overhead of DDMPD is significantly lower than Epidemic and Spray and Wait. When $n \longrightarrow \infty$, the routing overhead of DDMPD is zero compared with Epidemic's and Spray and Wait's routing overhead.

Therefore, when DDMPD has the same time complexity, it can have a lower routing overhead. In the following part, we used a simulation experiment to verify it.

\section{Simulation}

In order to evaluate the performance of the DDMPD, we use a simulation tool called ONE (Opportunistic Network Environment) [39] and to compare with three baseline approaches: SECM (status estimation and cache management algorithm) [13], ICMT (information cache management and data transmission algorithm) [5], and Spray and Wait routing algorithm [30]. The rationales of these algorithms are as follows:

(1) SECM: based on nodes, the algorithm can identify the surrounding neighbors to evaluate the transfer probability between nodes, thus caching data distribution adjustment. It also ensures that nodes have a high probability of transferring information first, thus achieving the purpose of cache adjustment. At the same time, the neighbor nodes cooperate to share the cache tasks of the nodes and effectively distribute data [13].

(2) ICMT: the algorithm evaluates the probability of nodes in the project based on the nodes that can identify the neighbors, thus evaluating the neighbors and ensuring the high project probability of nodes obtaining information preferentially, thus realizing the purpose of cache adjustment [5].

(3) Spray and Wait: the algorithm sprays a number of copies into the network and then waits till one of these nodes meets the destination [30]. The number of data copies of the algorithm will affect the performance, so we choose the number of copies to be 10 and 20, respectively, for the simulation.

Metric parameters used to evaluate these routing algorithms in the opportunistic network are as follows:

(1) Delivery ratio: that is, the probability of selecting a relay node during transmission.

(2) Overhead on average: this parameter shows the average overhead between two nodes when information is transmitted. 
(3) Energy consumption: it mainly records the energy consumption of nodes during transmission.

(4) End-to-end delay on average: indicates the average delay in passing information between two points.

A summary of the simulation parameters set in our experiments is presented as follows. The time of simulation is one to six hours, with a network area of $4500 \mathrm{~m} \times 3400 \mathrm{~m}$. The transmission pattern adopts broadcast. It involves 400 nodes. All nodes move randomly and move randomly at a speed of $0.5 \sim 1.5 \mathrm{~m} / \mathrm{s}$. The cache storage information of each node is as high as $5 \mathrm{Mb}$. The transmission pattern of nodes is the social model, the maximum transmission domain of each node is $10 \mathrm{~m}^{2}$, and the interval of sending data packet is 25-35 s with $250 \mathrm{~KB} / \mathrm{s}$ transmission speed. Also, each node's initial energy is 100 Joules, and it sends a data packet requiring $1 \mathrm{~J}$ energy consumption.

According to the data collected from the simulation report, the correlation between the time and the four measurement parameters is shown in Figures 7-10 below.

The relationship between the delivery ratio and the simulation time is shown in Figure 7. Among them, the lowest transmission rates are Spray and Wait routing algorithms $($ copy $=30)$ and SECM, which are $0.33-0.38$ and $0.37-0.41$, respectively. Because these two algorithms deliver information to nodes, the community by using the flooding method leads to mass information missing. Especially for Spray and Wait routing algorithms $($ copy $=30)$, the lower transmission rate is caused by copying a large amount of duplicate information. While the Spray and Wait routing algorithm $($ copy $=10)$ reduces the quantity of information copies, the delivery ratio increases to $0.42-0.47$. ICMT and DDMPD algorithm's delivery ratio is higher than 50\%. The ICMT algorithm controls the time interval of delivery information that improves the transmission and receiving of effective information, and its delivery ratio reached 0.54-0.58, which is $170 \%$ higher than that of the SECM algorithm. Due to the adoption of the DDMPD algorithm combining multisensing community and mobile node transmission, the transmission rate of the algorithm is effectively improved and is the highest among all algorithms, reaching $0.55-0.67$.

Figure 8 shows the association between routing overhead and stimulation time. In Figure 8, the routing overhead of the DDMPD algorithm is maintained between 115 to 118 , which is unaffected by time. With the increase in time, the number of nodes and communities participating in information transmission will also increase. Routing overhead can remain stable due to the increase in the number of shared information transmission nodes. Because the ICMT algorithm controls the frequency of delivery information, and its maximum routing overhead appeared in 2 hours, reaching 158 and then began to decrease, we can draw that the delay of algorithm is lower than other algorithms. However, the routing overhead of the Spray and Wait routing algorithm $($ copy $=30)$ is highest, showing that the algorithm has poor availability in the community model. Spray and Wait routing algorithms $($ COPY $=10)$ and SECM algorithms both have relatively close routing overhead

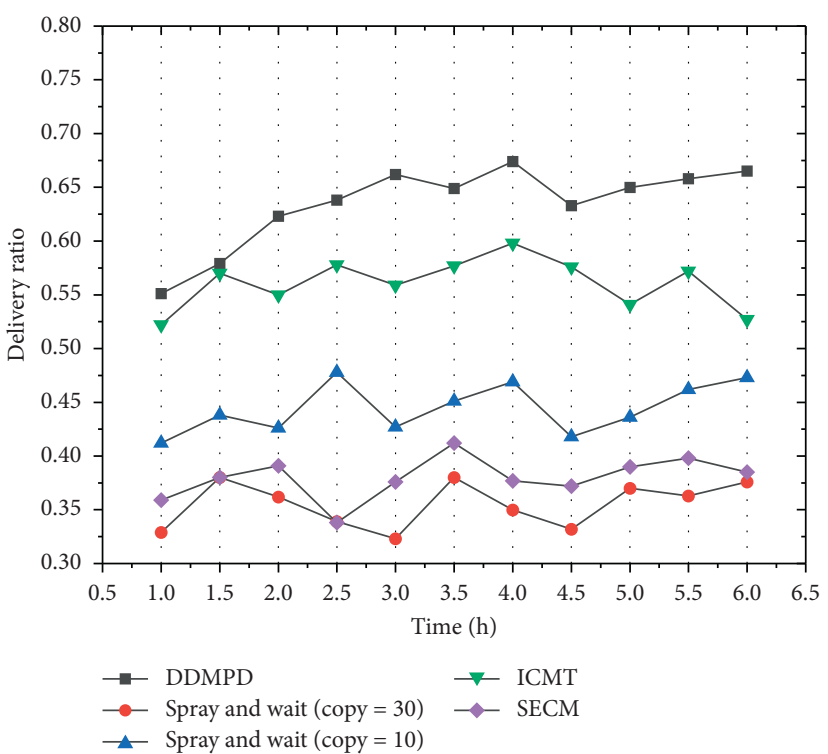

FIgURE 7: Relationship between delivery ratio and time.

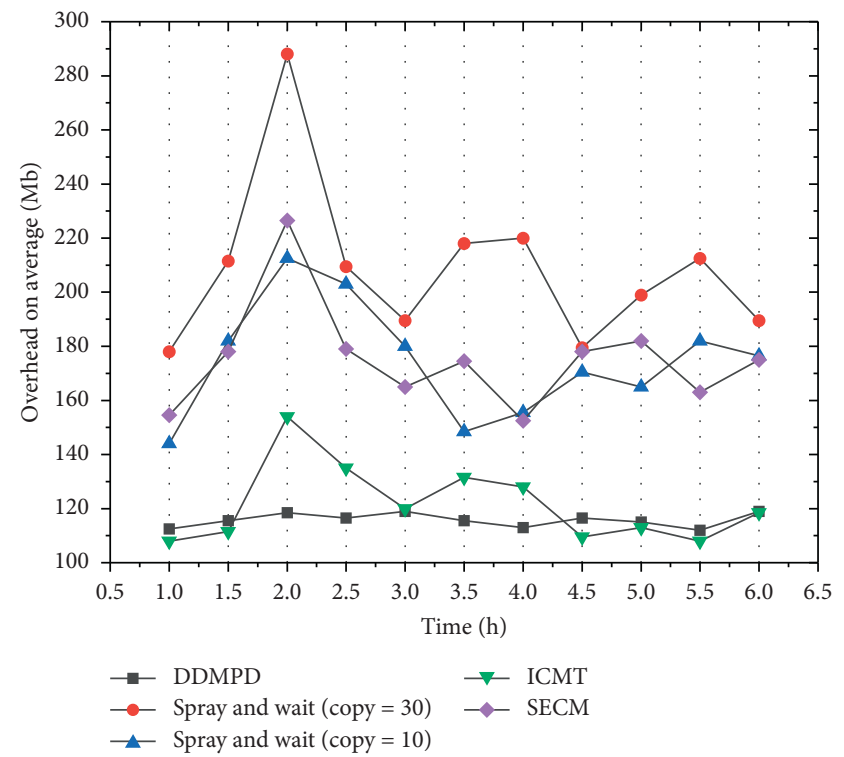

FIGURE 8: Relationship between average overhead and time.

ranging from 140 to 220 . It shows that the two algorithms are stable, but there is a local flooding phenomenon.

The relationship between energy consumption and stimulation time is shown in Figure 9. The energy consumption of these algorithms increases with time. However, the energy consumption of Spray and Wait routing algorithms is the highest because each node needs to transmit information through Spray. In particular, energy consumption exceeds 550 in 6 hours. The SECM algorithm uses the method of encounter transmission and copy information by single duplication, and it is better than the Spray and Wait routing algorithm in energy optimizing. In the process of information transmission, several communities bear the energy consumption and extend the information transmission time of nodes, thus reducing the number of 


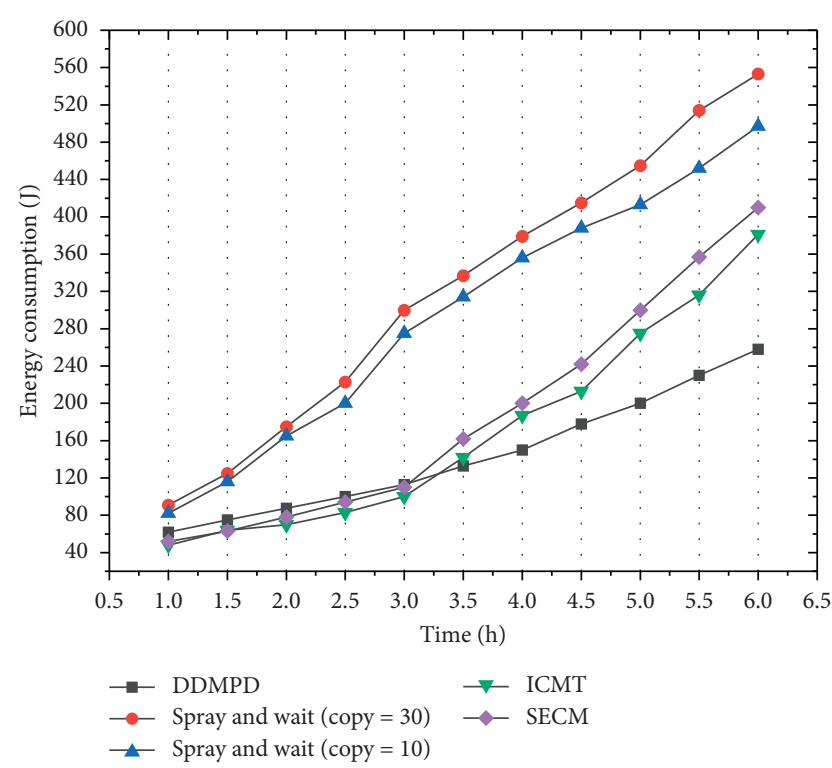

FIgURE 9: Relationship between energy consumption and time.

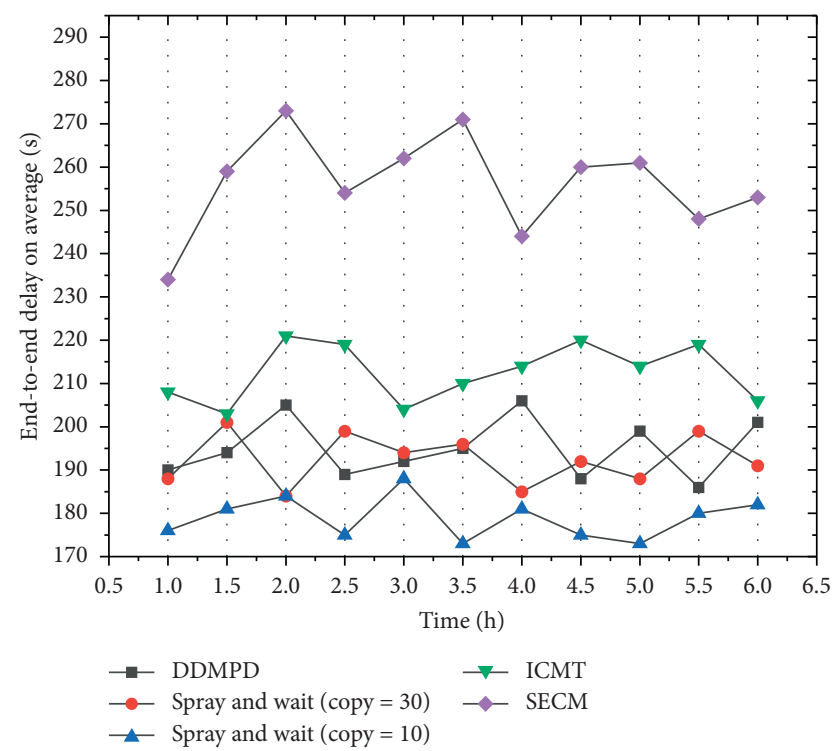

Figure 10: Relationship between end-to-end delay on average and time.

information transmission times to source nodes. The energy consumption of the DDMPD algorithm is less than other algorithms. It has $65 \%$ of SECM algorithm's and only $45 \%$ of Spray and Wait routing algorithm's.

Figure 10 shows the relationship between mean transmission delay and time. The SECM algorithm adopts the method of encounter delivery that mass copied information transmits on the community and causes a high transmission delay that is the average delay time of the SECM algorithm exceeds 260. Although the time interval of information transmission is controlled by the ICMT algorithm, there is still a high transmission delay, reaching 210 . The results show that the SECM algorithm is a high delay algorithm in the community model and has an important impact on information transmission. Because the information delivery between communities occupies mass of network resource and causes the delay, therefore, the delay of the DDMPD algorithm is higher than Spray and Wait routing algorithm's $($ copy $=10)$, averagely achieving 195 . Because the transmission delay of Spray and Wait routing algorithms is low, it can indicate that their information diffusion capability is strong.

To sum up, we can conclude that the DDMPD algorithm is superior to other algorithms in terms of transmission rate, routing overhead, and energy consumption, but its time delay is higher than Spray and Wait routing algorithm's. In a real environment, the DDMPD is better than other algorithms in long-time information transmission.

In social networks, node cache is an important indicator, and the transmission efficiency of the algorithm will be directly affected by it. Therefore, we combine cache with four metric parameters, and the following are the specific experimental results (Figures 11-14).

The correlation between delivery ratio and cache is shown in Figure 11. The figure shows that the algorithm with the highest delivery rate is the DDMPD algorithm, reaching 0.58-0.83. Because the DDMPD algorithm uses the method of combining multiple perception community and node mobile delivery in the condition of increasing node cache that the delivery ratio of the algorithm is significant. Because Spray and Wait routing algorithms $($ copy $=30)$ use flooding to transmit information at community nodes, a large amount of information is lost and the transmission rate is the lowest, only $0.32-0.47$. ICMT algorithm and Spray and Wait routing algorithm (copy $=10)$ improve the condition of information transmission and increased the delivery ratio of algorithms, more than $50 \%$.

The relationship between routing overhead and cache is shown in Figure 12. In Figure 12, four algorithms with increasing cache make their routing overhead significantly lower. The routing overhead of the DDMPD algorithm drops from 210 from 23; the routing overhead of the Spray and Wait routing algorithm $($ copy $=10)$ falls to 50 from 280; the routing overhead of the Spray and Wait routing algorithm $($ copy $=10)$ drops from 330 down to 80 . The above three algorithms' drop is notable, and it shows a larger node cache that a lower node overhead. Compared with the other three algorithms, Spray and Wait routing algorithms $($ copy $=30)$ and SECM algorithms have very low drop rates. The above results show that increasing the routing overhead of nodes in the community can be achieved by increasing the node cache.

Figure 13 shows the relationship between energy consumption and cache. When the cache is increasing, the energy consumption of algorithms grows sharply, except the DDMPD algorithm's energy being always maintained at around 43. The DDMPD algorithm uses the method of information delivery provided by the community that reduces energy consumption significantly. Spray and Wait routing algorithms consume the most energy. Each node uses spray to transmit information to all neighbors in the community, thus consuming a lot of energy. Especially in the case of copy $=30$, when the cache reaches $40 \mathrm{Mb}$, energy 


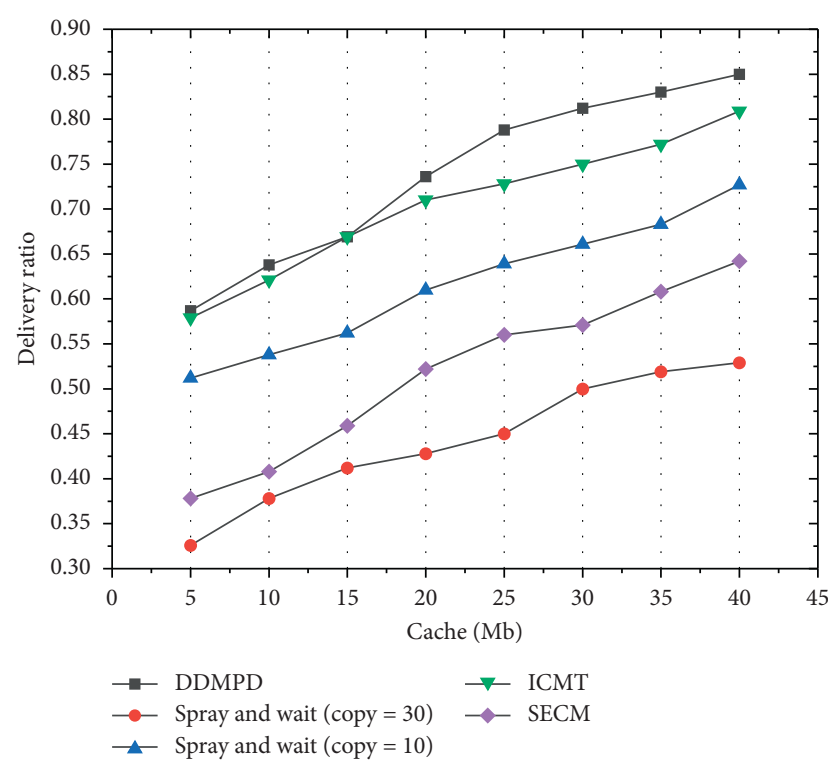

Figure 11: Relationship between the delivery ratio and cache.

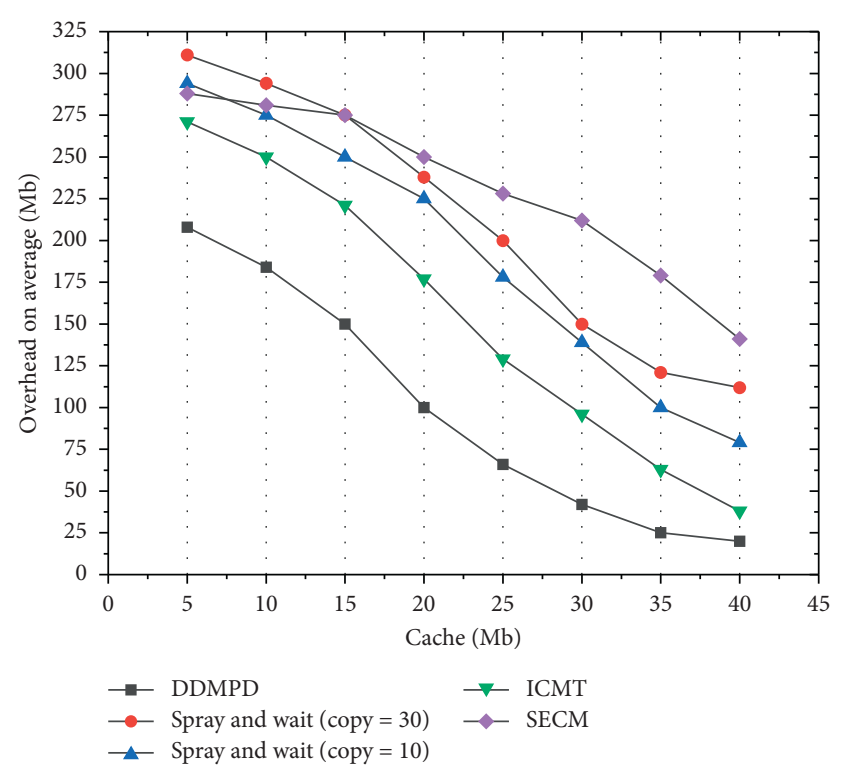

Figure 12: Relationship between average overhead and cache.

consumption exceeds 280 . The SECM algorithm adopts the encounter transmission mode and replicates information through single replication. Compared with Spray and Wait routing algorithms, its energy optimization effect is better.

Figure 14 shows the correlation between the average delivery delay and the cache. The node delivery delay declines with the increasing cache, and the average delay of the SECM algorithm drops from 200 to 90; the ICMT algorithm controls the time interval of information transmission, and its average delay slumps from 175 to 56. DDMPD and Spray and Wait routing algorithms with similar transmission delays $($ copy $=30)$ all dropped from 100 to less than 40 . The results show that increasing the network transmission delay can increase the node cache.

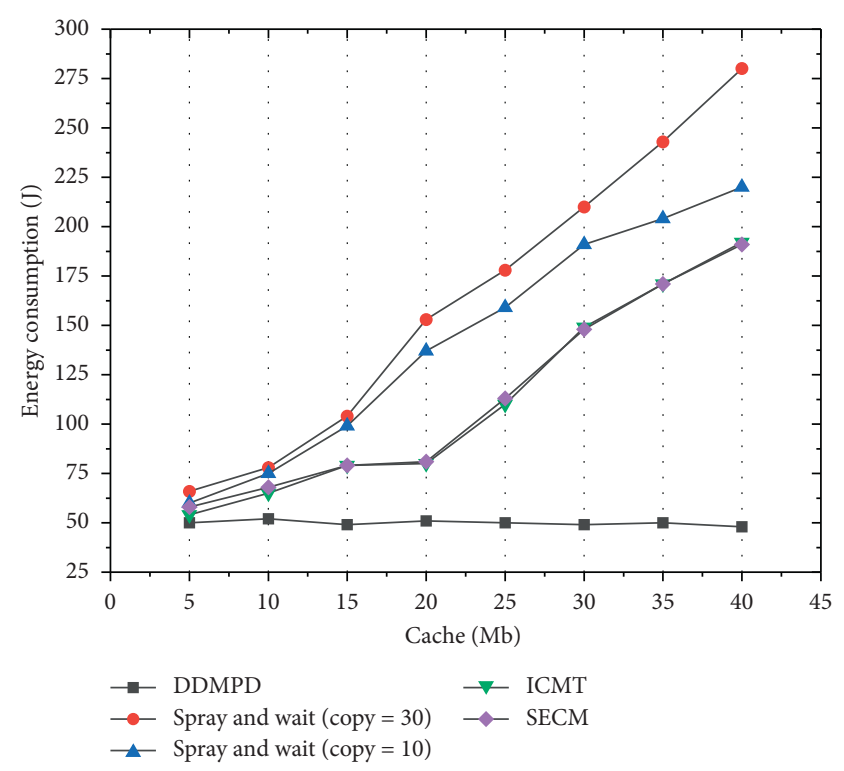

FIGURE 13: Relationship between energy consumption and cache.

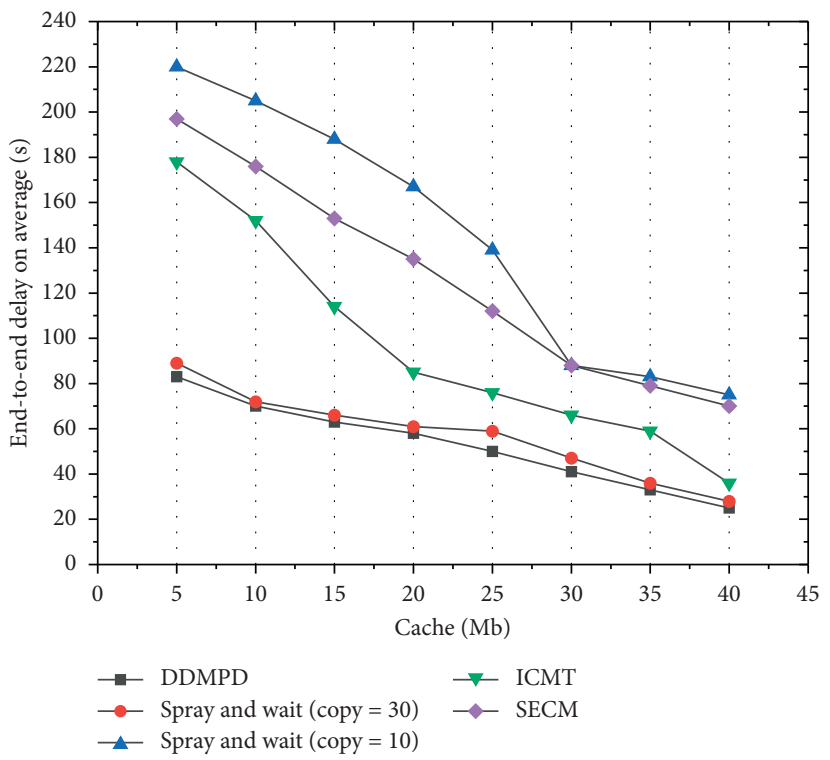

FIgURE 14: Relationship between end-to-end delay on average and cache.

In addition to the above four measurement parameters to be considered, there may be a variety of information transfer methods in the actual environment. Therefore, the use of different moving methods in the simulation environment also needs to be considered. We select three mobile models to confirm the DDMPD's performance, and they are SPMBM (Shortest Path Map-Based Movement), random way point (RWP), and random walk (RM) models [39].

The delivery ratio of the DDMPD algorithm in different mobile models is shown in Figure 15. In figure DDMPD, the highest delivery ratio is the SPMBM model, reaching 0.59 in 4 hours. The delivery ratio of the RWP model can reach a peak of 0.62 in 4 hours, while the delivery ratio of the RM model can reach a peak of 0.62 in 6 hours. The overall 


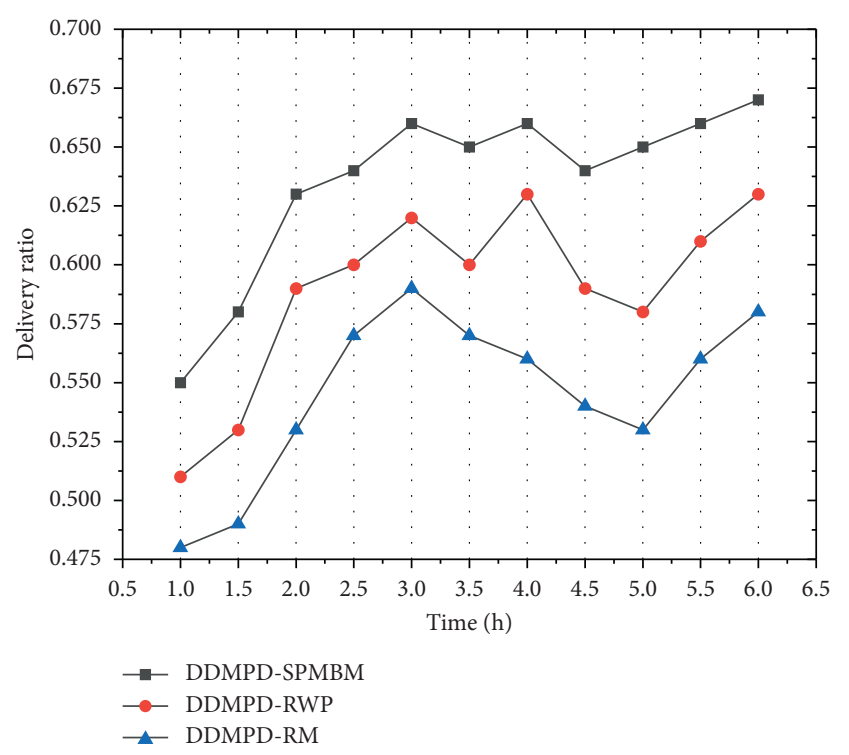

FIGURE 15: Relationship between delivery ratio and time in three mobile models.

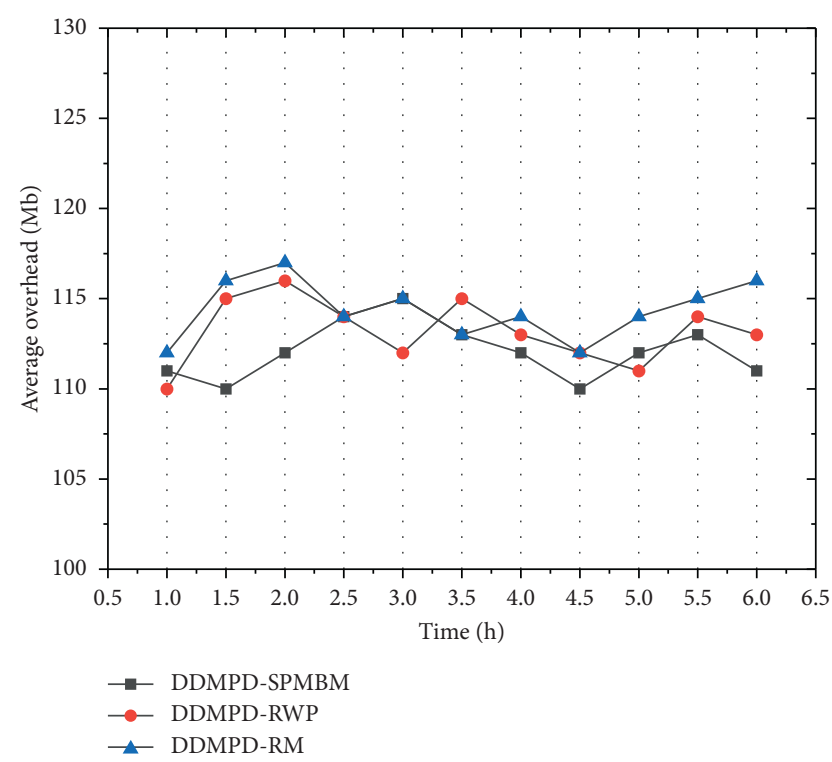

FIGURE 16: Relationship between average delay and time in three mobile models.

performance and maximum delivery rate are the SPBM model, and the delivery rate of the RWP model is higher than the RM model.

Figure 16 shows the DDMPD algorithm's routing overhead in three kinds of mobile models. As shown in Figure 16, the DDMPD algorithm's routing overhead is little affected, and the routing overhead range of three models is between 110 and 120. This is because of the process of node movement and information transfer, and the community forms the task of sharing information with mass nodes based on the process of information transfer and node reconstruction.

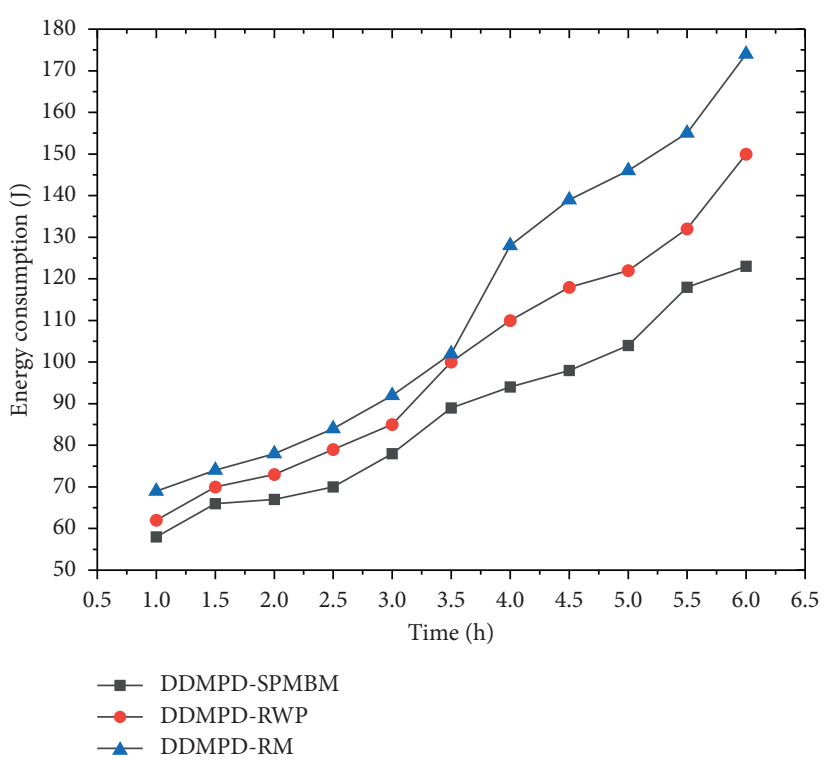

FIGURE 17: Relationship between energy consumption and time in three mobile models.

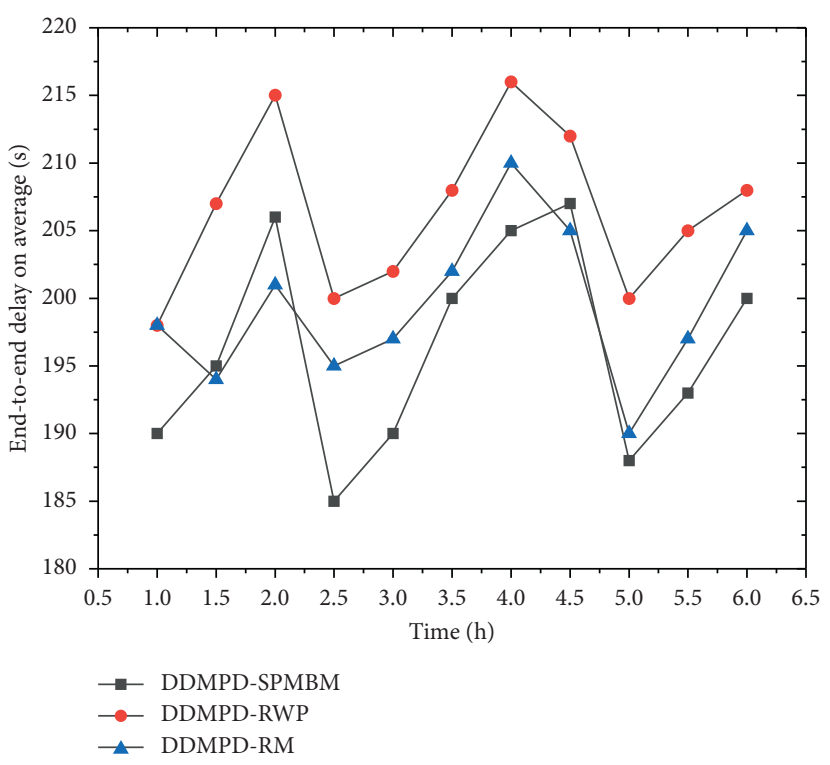

FIGURE 18: Relationship between energy consumption and time in three mobile models.

Figure 17 shows the energy consumption of DDMPD in different mobile models. There is little difference in energy consumption among the three models. The results show that the DDMPD algorithm has stable node information transmission performance and a large amount of energy will not be consumed when the model changes.

Figure 18 shows the average delay of the DDMPD in 3 mobile models. For all models between 185 and 220, the models of delay, maximum delay, and minimum delay are RM and SPBM, respectively. The results show that the SPMBM model is very effective in transmitting information. 


\section{Conclusion}

An effective data transmission scheme designed in this paper solves the problem of excessive energy consumption of source nodes and compares and analyzes the performance of classical opportunistic network algorithms. The experiment results show that this algorithm has a good ability of transmission, and it has an excellent performance in three different mobile models, indicating the algorithm is steady in different environments. In opportunistic social networks, this scheme can reduce the energy consumption of nodes, prolong the network life cycle, and greatly improve the data transmission efficiency.

5G terminals have more severe energy consumption problems than the previous generation of communication standards, which will become one of the important reasons affecting the user experience of 5G networks. Therefore, reducing the energy consumption of source nodes and relay nodes will be the focus of future work. In order to reduce the energy consumption of the source node, it is necessary to improve the efficiency of large-capacity data transmission between nodes.

\section{Data Availability}

The data used to support the findings of this study are currently under embargo, while the research findings are commercialized. Requests for data, 12 months after publication of this article, will be considered by the corresponding author.

\section{Conflicts of Interest}

The authors declare that they have no conflicts of interest.

\section{Acknowledgments}

This work was supported by the National Natural Science Foundation of China (61672540) and Hunan Provincial Natural Science Foundation of China (2018JJ3299 and 2018JJ3682).

\section{References}

[1] M. Ammar, G. Russello, and B. Crispo, "Internet of things: a survey on the security of iot frameworks," Journal of Information Security and Applications, vol. 38, pp. 8-27, 2018.

[2] J. Wu, M. Z. Chen, and M. Zhao, "Community recombination and duplication node traverse algorithm in opportunistic social networks," Peer-to-Peer Networking and Applications, pp. 1-8, 2020.

[3] X. Zhang, Y. Yuan, Z. Zhou, S. Li, L. Qi, and D. Puthal, "Intrusion detection and prevention in cloud, fog, and internet of things," Security and Communication Networks, vol. 2019, Article ID 4529757, 4 pages, 2019.

[4] R.-C. Marin, R.-I. Ciobanu, C. Dobre, C. X. Mavromoustakis, and G. Mastorakis, "A context-aware collaborative model for smartphone energy efficiency over $5 \mathrm{~g}$ wireless networks," Computer Networks, vol. 129, pp. 352-362, 2017.

[5] J. Wu, Z. Chen, and M. Zhao, "Information cache management and data transmission algorithm in opportunistic social networks," Wireless Networks, vol. 25, no. 6, pp. 2977-2988, 2019.

[6] J. Wu, Z. Chen, and M. Zhao, "An efficient data packet iteration and transmission algorithm in opportunistic social networks," Journal of Ambient Intelligence and Humanized Computing, pp. 1-17, 2019.

[7] L. Gupta, R. Jain, and G. Vaszkun, "Survey of important issues in uav communication networks," IEEE Communications Surveys \& Tutorials, vol. 18, no. 2, pp. 1123-1152, 2015.

[8] X. Xu, Y. Xue, L. Qi et al., "An edge computing-enabled computation offloading method with privacy preservation for internet of connected vehicles," Future Generation Computer Systems, vol. 96, pp. 89-100, 2019.

[9] W. Gong, L. Qi, and Y. Xu, "Privacy-aware multidimensional mobile service quality prediction and recommendation in distributed fog environment," Wireless Communications and Mobile Computing, vol. 2018, Article ID 3075849, 8 pages, 2018.

[10] W. Dou, W. Tang, S. Li, S. Yu, and K.-K. Raymond Choo, “A heuristic line piloting method to disclose malicious taxicab driver's privacy over GPS big data," Information Sciences, vol. 483, pp. 247-261, 2019.

[11] J. Wu and Z. Chen, "Sensor communication area and node extend routing algorithm in opportunistic networks," Peer-toPeer Networking and Applications, vol. 11, no. 1, pp. 90-100, 2018.

[12] J. Wu, G. Yu, and P. Guan, "Interest characteristic probability predicted method in social opportunistic networks," IEEE Access, vol. 7, pp. 59002-59012, 2019.

[13] J. Wu, Z. Chen, and M. Zhao, "SECM: status estimation and cache management algorithm in opportunistic networks," The Journal of Supercomputing, vol. 75, no. 5, pp. 2629-2647, 2019.

[14] L. Atzori, A. Iera, G. Morabito, and M. Nitti, "The Social Internet of Things (SIoT) - when social networks meet the Internet of Things: concept, architecture and network characterization," Computer Networks, vol. 56, no. 16, pp. 35943608, 2012.

[15] X. Lan, Q. Chen, X. Tang, and L. Cai, "Achievable rate region of the buffer-aided two-way energy harvesting relay network," IEEE Transactions on Vehicular Technology, vol. 67, no. 11, pp. 11127-11142, 2018.

[16] L. Qi, Y. Chen, Y. Yuan, S. Fu, X. Zhang, and X. Xu, "A QoSaware virtual machine scheduling method for energy conservation in cloud-based cyber-physical systems," World Wide Web, pp. 1-23, 2019.

[17] L. Qi, X. Zhang, W. Dou, C. Hu, C. Yang, and J. Chen, “A twostage locality-sensitive hashing based approach for privacypreserving mobile service recommendation in cross-platform edge environment," Future Generation Computer Systems, vol. 88 , pp. $636-643,2018$.

[18] Y. Xu, L. Qi, W. Dou, and J. Yu, "Privacy-preserving and scalable service recommendation based on simhash in a distributed cloud environment," Complexity, vol. 2017, Article ID 3437854, 9 pages, 2017.

[19] X. Zhang, J. Jang-Jaccard, L. Qi, M. Z. Bhuiyan, and C. Liu, "Privacy issues in big data mining infrastructure, platforms, and applications," Security and Communication Networks, vol. 2018, Article ID 6238607, 3 pages, 2018.

[20] H. Liu, H. Kou, C. Yan, and L. Qi, "Link prediction in paper citation network to construct paper correlation graph," EURASIP Journal on Wireless Communications and Networking, vol. 2019, no. 1, pp. 1-12, 2019.

[21] W. Dou, X. Xu, S. Meng et al., "An energy-aware virtual machine scheduling method for service qos enhancement in 
clouds over big data," Concurrency and Computation: Practice and Experience, vol. 29, no. 14, Article ID e3909, 2017.

[22] Y. Yuan, Y.-S. Ong, A. Gupta, P. S. Tan, and H. Xu, "Evolutionary multitasking in permutation-based combinatorial optimization problems: realization with TSP, QAP, LOP, and JSP," in Proceedings of the 2016 IEEE Region 10 Conference (TENCON), pp. 3157-3164, IEEE, Singapore, November 2016.

[23] Y. Yuan, Y.-S. Ong, A. Gupta, and H. Xu, "Objective reduction in many-objective optimization: evolutionary multiobjective approaches and comprehensive analysis," IEEE Transactions on Evolutionary Computation, vol. 22, no. 2, pp. 189-210, 2017.

[24] J. Wu, Z. Chen, and M. Zhao, "Weight distribution and community reconstitution based on communities communications in social opportunistic networks," Peer-to-Peer Networking and Applications, vol. 12, no. 1, pp. 158-166, 2019.

[25] A. Vahdat and D. Becker, "Epidemic Routing for Partially Connected Ad Hoc Networks," Handbook of Systemic Autoimmune Diseases, Elsevier, Amsterdam, Netherlands, 2000.

[26] X. Lu and P. Hui, "An energy-efficient n-epidemic routing protocol for delay tolerant networks," in Proceedings of the 2010 IEEE Fifth International Conference on Networking, Architecture, and Storage, pp. 341-347, IEEE, Macau, China, July 2010.

[27] P. Guan and J. Wu, "Effective data communication based on social community in social opportunistic networks," IEEE ACCESS, vol. 7, no. 1, pp. 12405-12414, 2019.

[28] J. Burgess, B. Gallagher, D. D. Jensen, and B. N. Levine, "Maxprop: routing for vehicle-based disruption-tolerant networks," in Proceedings of the IEEE INFOCOM 2006: 25TH IEEE International Conference on Computer Communications, vol. 6, Barcelona, Spain, April 2006.

[29] M. Das, S. Sarkar, and S. M. A. Iqbal, "TTL based maxprop routing protocol," in Proceedings of the 2016 19th International Conference on Computer and Information Technology (ICCIT), pp. 7-12, IEEE, Dhaka, Bangladesh, December 2016.

[30] T. Spyropoulos, K. Psounis, and C. S. Raghavendra, "Spray and wait: an efficient routing scheme for intermittently connected mobile networks," in Proceedings of the 2005 ACM SIGCOMM Workshop on Delay-Tolerant Networking, pp. 252-259, ACM, Philadelphia, PA, USA, August 2005.

[31] G. Wang, B. Wang, and Y. Gao, "Dynamic spray and wait routing algorithm with quality of node in delay tolerant network," in Proceedings of the 2010 International Conference on Communications and Mobile Computing, vol. 3, pp. 452456, IEEE, Shenzhen, China, April 2010.

[32] J. Guan, Q. Chu, and I. You, "The social relationship based adaptive multi-spray-and-wait routing algorithm for disruption tolerant network," Mobile Information Systems, vol. 2017, Article ID 1819495, 13 pages, 2017.

[33] A. Lindgren, A. Doria, and O. Schelén, "Probabilistic routing in intermittently connected networks," in Proceedings of the International Workshop on Service Assurance with Partial and Intermittent Resources, pp. 239-254, Springer, Fortaleza, Brazil, August 2004.

[34] J. Xue, J. Li, Y. Cao, and J. Fang, "Advanced prophet routing in delay tolerant network," in Proceedings of the 2009 International Conference on Communication Software and Networks, pp. 411-413, IEEE, Macau, China, February 2009.

[35] S. D. Han and Y. W. Chung, "An improved prophet routing protocol in delay tolerant network," The Scientific World Journal, vol. 2015, Article ID 623090, 7 pages, 2015.
[36] M. Musolesi and C. Mascolo, "CAR: context-aware adaptive routing for delay-tolerant mobile networks," IEEE Transactions on Mobile Computing, vol. 8, no. 2, pp. 246-260, 2008.

[37] J. Niu, J. Guo, Q. Cai, N. Sadeh, and S. Guo, "Predict and spread: an efficient routing algorithm for opportunistic networking," in Proceedings of the 2011 IEEE Wireless Communications and Networking Conference, pp. 498-503, IEEE, Cancun, Mexico, March 2011.

[38] M. E. J. Newman, "Analysis of weighted networks," Physical Review E, vol. 70, no. 5, Article ID 056131, 2004.

[39] A. Keranen, "Opportunistic network environment simulator," Special Assignment Report, Helsinki University of Technology, Department of Communications and Networking, Espoo, Finland, 2008. 